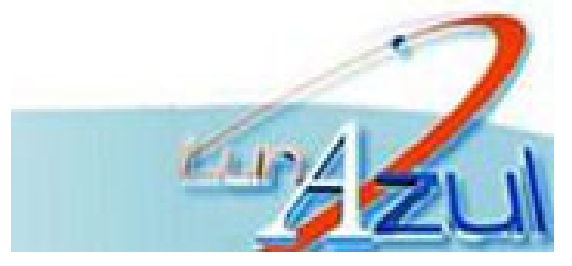

Revista Luna Azul

E-ISSN: 1909-2474

lesga@une.net.co

Universidad de Caldas

Colombia

Zarazúa, José-Alberto; Mazabel, Davison; Moncayo-Estrada, Rodrigo; Rendón-Medel, Roberto EVALUACIÓN DE LA SUSTENTABILIDAD EN ESPACIOS RURALES RELACIONADOS CON EL ECOTURISMO. ESTUDIO DE CASO EN LA SELVA LACANDONA, CHIAPAS, MÉXICO

Revista Luna Azul, núm. 40, enero-junio, 2015, pp. 47-68

Universidad de Caldas

Manizales, Colombia

Disponible en: http://www.redalyc.org/articulo.oa?id=321733015005

- Cómo citar el artículo

Número completo

- Más información del artículo

- Página de la revista en redalyc.org

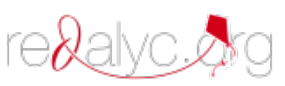

Sistema de Información Científica

Red de Revistas Científicas de América Latina, el Caribe, España y Portugal Proyecto académico sin fines de lucro, desarrollado bajo la iniciativa de acceso abierto 


\title{
EVALUACIÓN DE LA SUSTENTABILIDAD EN ESPACIOS RURALES RELACIONADOS CON EL ECOTURISMO. ESTUDIO DE CASO EN LA SELVA LACANDONA, CHIAPAS, MÉXICO ${ }^{1}$
}

\author{
José-Alberto Zarazúa ${ }^{2}$ \\ Davison Mazabel ${ }^{3}$ \\ Rodrigo Moncayo-Estrada ${ }^{4}$ \\ Roberto Rendón-Medel ${ }^{5}$
}

Recibido el 3 de abril de 2014, aprobado el 16 de agosto de 2014, actualizado el 1 noviembre de 2014

\section{DOI: 10.17151/luaz.2015.40.5}

\section{RESUMEN}

Esta investigación realiza una evaluación ex-post de la sustentabilidad en espacios rurales relacionados con el ecoturismo, centrada en un estudio comparativo de tres empresas enclavadas en la Reserva de la Biosfera Montes Azules (REBIMA) y una empresa más ubicada fuera de ella, todas en el municipio de Ocosingo, Chiapas, México. $75 \%$ de los entrevistados son hombres con edad promedio de 36 años, siete años de escolaridad y 1,9 hectáreas. La principal fuente de ingresos es el ecoturismo, que presenta una relación beneficio-costo venida a menos de 2,7 a 1,7, entre 2007 y 2011, respectivamente. La evidencia indica un desequilibrio en el acceso a subsidios, donde la pertenencia étnica desempeña un rol trascendental.

\section{PALABRAS CLAVE}

Ecoturismo; Evaluación de la Sustentabilidad; Área Natural Protegida; Reserva de la Biósfera; Selva Lacandona, México.

\section{ASSESSMENT OF THE SUSTAINABILITY IN RURAL SPACES RELATED TO ECOTOURISM. CASE STUDY IN LACANDON JUNGLE, CHIAPAS, MEXICO}

\begin{abstract}
This research performs an ex-post evaluation of the sustainability in rural spaces related to ecotourism, focused on a comparative study of three companies located in the Montes Azules Biosphere Reserve (MABR) and another company located outside, all of them in the town of Ocosingo, Chiapas, México. $75 \%$ of respondents are male with an average age of 36 years, seven years of schooling and 1,9 hectares. The main source of income is ecotourism, which presents a cost-benefit relationship that has ran down from 2,7 to 1,7 between 2007 and 2011, respectively. The evidence indicates an imbalance in the access to subsidies, where the ethnical ownership plays a transcendental role.
\end{abstract}

\section{KEY WORDS}


Ecotourism; Evaluation of sustainability; Natural Protected Area; Biosphere Reserve; Lacandon Jungle, Mexico.

\section{INTRODUCCIÓN}

La actividad turística, entendida como las actuaciones de las personas que viajan y permanecen en territorios diferentes al habitual, durante un año o menos de manera consecutiva con fines de goce del tiempo libre, arreglo de negocios u otros (WTTC, 2012a, p. 11), constituye una atractiva fuente de ingresos y de recursos para el desarrollo, especialmente en economías emergentes (Hernández, López y Pons, 2006, p. 7; UN, 2010).

El Consejo Mundial de Turismo y Viajes (WTTC por sus siglas en inglés) estimó que en Latinoamérica la contribución directa al PIB alcanzó 3,2\% (USD\$133,8 mil millones) y la contribución total 8,6\% del PIB (USD\$ 364,3 mil millones) para 2011 (WTTC, 2012b, p. 1), en tanto que la generación de empleos totales fue de $7,7 \%$ de los empleos generados (15'282.500), durante 2011. Como era de esperarse, México no permaneció al margen y las estadísticas indican que la contribución directa al PIB alcanzó 5,6\% (MX \$791,8 mil millones), la contribución total ascendió a 12,4\% del PIB (MX $\$ 1.763,6$ mil millones) y el aporte en la generación de empleos fue del 13,7\% del total generado durante 2011 (6’347.500) (WTTC, 2012c, p. 1). Otras economías en las que el turismo realiza un aporte sobresaliente al PIB son Costa Rica (4,8\% de contribución directa y $12,3 \%$ de contribución total) (WTTC, 2012d, p. 1) y Argentina $(3,8 \%$ de contribución directa y $10,5 \%$ de contribución total) (WTTC, 2012e, p. 1). ${ }^{6}$

En este contexto, la evaluación del grado de sustentabilidad en los espacios rurales, es de particular interés, máxime cuando en dichos territorios se desarrollan actividades ecoturísticas. Jules (1995, p. 1248) indica que es relativamente sencillo describir los objetivos de la sustentabilidad, que intentar definirla de forma precisa, no obstante, se reconoce que la sustentabilidad es un concepto dinámico que aborda un devenir continuo, razón por la que en el diseño y la difusión de sistemas complejos (económicos, ecológicos, de recursos naturales, entre otros) es requerido contemplar perspectivas de largo plazo y comprender/articular las distintas dinámicas socioambientales en las dimensiones tiempo y espacio (Galván, Masera y López, 2008, pp. 42-43). Masera et al. (2008, p. 17) refieren que además de tratarse de un concepto dinámico, es multidimensional y específico a un contexto socioambiental y espacio-temporal determinado.

Galván et al. (2008, p. 42) mencionan que existen tres grandes tendencias en torno a la evaluación de la sustentabilidad, mismas que las agrupa de la forma siguiente: a) Aquellas centradas en la generación de listas de indicadores de sustentabilidad, enfocados en aspectos ambientales, económicos y, en menor medida, sociales e institucionales, que en general, carecen de una base teórica para 
la selección de indicadores específicos, y no es posible aplicar los indicadores a contextos diversos. b) Otras avocadas a metodologías de evaluación basadas en la determinación de índices de sustentabilidad, en las cuales se agrega o sintetiza la información de los indicadores en un solo valor numérico, mismas que no ofrecen un marco analítico adecuado para la derivación de indicadores, además de que su construcción requiere decisiones arbitrarias en cuanto a la selección, ponderación y agregación de indicadores. $Y$ por último, c) los métodos que integran los marcos de evaluación, es decir, propuestas metodológicas flexibles que permiten guiar el proceso de evaluación mediante diferentes etapas, y que se formulan a partir de atributos u objetivos generales que son aplicables en diferentes situaciones y sistemas de manejo, y que sirven de guía para derivar criterios e indicadores más específicos.

La presente investigación se circunscribe en este último grupo de metodologías, se apoya en las investigaciones de Rendón (2004), De Camino y Müller (1993) y OCDE (1993). Algunos de los aportes del trabajo son los siguientes: 1) ofrece un marco analítico para evaluar y proporcionar el debido seguimiento a la sustentabilidad en sistemas no agrícolas, y particularmente, relacionado con el ecoturismo en espacios rurales; 2) se identificaron seis ejes temáticos que permitieron priorizar y seleccionar un conjunto de 19 variables que coadyuvan al monitoreo de un sistema no agrícola, y 3) aporta elementos que contribuyen al proceso de toma de decisiones con base en información, que puede ser fundamento de un sistema de evaluación y seguimiento institucional y/o empresarial.

Esta investigación realiza una propuesta metodológica en torno a un marco de evaluación ex-post de la sustentabilidad en espacios rurales relacionados con el ecoturismo. Asimismo, teniendo en cuenta la necesidad de evaluar la sustentabilidad en sistemas previo a su implementación (evaluaciones ex-ante), se procuró "reconstruir" un escenario en tiempo cero (2007) que proporcionara información y permitiera comparar el grado de la sustentabilidad en el tiempo actual (2011). El objetivo de la investigación fue diseñar un índice de sustentabilidad ecoturística basado en la integración de seis ejes: económicos, sociales, tecnológicos, institucionales, medioambientales y del entorno, que integra a un total de 19 variables, mismo que permitió realizar un estudio comparativo con tres empresas ecoturísticas enclavadas en la Reserva de la Biosfera Montes Azules (REBIMA) y una empresa más ubicada fuera de ella, establecidas todas en el municipio de Ocosingo, Chiapas, México.

En este trabajo, se concibe al ecoturismo como una modalidad del turismo alternativo, que se ofrece a turistas que gustan de actividades recreativas de apreciación y conocimiento de la naturaleza a través de la interacción con la misma, y que implica, un viaje responsable que incide además en el bienestar de habitantes locales (TIES ${ }^{7}, 1990 ;$ SECTUR $^{8}, 2004$, p. 7). Se parte de una definición del espacio rural, aplicable a las localidades de estudio de la Selva Lacandona en el estado de Chiapas, México (Ivars, 2000, p. 60): i) predominio de paisajes poco transformados, ii) presencia de núcleos poblacionales pequeños, iii) aislamiento geográfico por déficit de infraestructura carretera y de telecomunicaciones, iv) homogeneidad en los hábitos y cambios sociales, como aquellos 
que plantea el cambio en el patrón de consumo de alimentos al pasar de tortillas recién hechas (a mano) por tortillas fabricadas en máquinas, y v) pervivencia de manifestaciones culturales ancestrales.

Para el caso particular del área de estudio es importante señalar además que el poblamiento tardío - predominante a partir de la segunda mitad del siglo XX - y la concurrencia de diferentes culturas en la zona, distintas lenguas y diversas experiencias, según los lugares de origen, ha generado una dinámica sociocultural muy particular, no exenta de dificultades y riesgos.

Precisamente por tratarse de espacios rurales con presencia de población indígena, y una relativamente reciente colonización, que impuso procesos de transformación socioeconómica y ambiental complejos, resulta pertinente realizar estudios que permitan valorar los impactos, en términos de la sustentabilidad, de actividades económicas como el ecoturismo, que no solo se presentan como una alternativa sino que también parecen prometer beneficios para el desarrollo de estos territorios.

\section{CONTEXTO SOCIOECONÓMICO}

En la zona que comprende la reserva y el área de influencia de esta, existen una serie de condicionantes antrópicas, entre ellas el contar con grupos de población indígena -choles, tzeltales, lacandonesque conforman un mosaico socioeconómico y cultural muy particular, con características especiales y que están influidas en el presente por sucesivas oleadas de inmigración procedentes principalmente de los Altos y el Norte de Chiapas, así como de otros estados del país.

Dicho proceso, aceleró la incorporación de la Selva Lacandona a la producción agropecuaria y forestal, con el consecuente cambio en el uso del suelo y la drástica disminución de la superficie forestal. Lo que aunado a la apertura de la carretera fronteriza, la exploración y explotación petroleras en la selva han acentuado este fenómeno (INE, 2000).

Los habitantes más antiguos de la REBIMA son los lacandones. Hasta mediados de la década de los sesenta, la inmensidad del bosque tropical protegió a los mayas lacandones de los muchos cambios que sufrieron otros grupos indígenas de México, por lo que lograron conservar muchos de los métodos de uso de los recursos naturales especialmente adecuados al ecosistema tropical.

La región ha sido caracterizada como una zona de economía campesina de autoconsumo con una producción mercantil complementaria basada en el cultivo del chile y café, cría de cerdos, becerros, recolección de palmas y explotación forestal, es decir que se le ha visto como: 
[...] una unidad bien diferenciada de otras regiones por las condiciones del sustrato natural para la producción, las características tecnológicas del manejo de los recursos, el objetivo y destino de la producción y el tipo de relaciones sociales y económicas que se establecen para el desarrollo y para la producción. (INE, 2000, p. 46)

Aunque otros autores (Leyva y Ascencio, 1996), han enfatizado que no se trata de una región encerrada en sí misma y contenida en los límites del espacio natural, y que incluso las relaciones comerciales se dan con Holanda y Estados Unidos hacia donde exportan café y con otras ciudades del país a donde llega carne de los becerros criados en la selva lacandona.

Pese a lo anterior, se ha señalado que:

[...] la mayor parte de los pobladores no disponen de los recursos ni de los conocimientos necesarios para llevar a cabo cultivos alternativos o para beneficiarse de un aprovechamiento sostenido de las plantas y animales tropicales. Por otro lado, es evidente y alarmante el proceso de desculturización y la pérdida de conocimientos tradicionales sobre el manejo de los recursos naturales. (INE, 2000, p. 45)

La transición que ha vivido el territorio que aquí estudiamos puede resumirse señalando, de manera paradójica, que uno de los cinco focos rojos de la entidad chiapaneca durante las últimas décadas lo constituyeron las invasiones a la Comunidad Lacandona y Reserva Integral de la Biosfera Montes Azules, lo que derivó en muchos conflictos agrarios (Reyes, 2004). Pero al mismo tiempo, algunos estudios realizados sobre Reservas de la Biosfera, destacan los beneficios que ha generado la actividad ecoturística organizada por comunidades indígenas en fragmentos de selva en la periferia de la reserva de Montes Azules (Navarrete y Halffter, 2008; Halffter, 2011).

\section{METODOLOGÍA}

La investigación se realizó en la Reserva de la Biosfera Montes Azules (REBIMA), también conocida como Selva Lacandona, establecida en los municipios de Ocosingo y Maravilla Tenejapa en el estado de Chiapas: i) es la principal selva tropical de México, con una superficie de 331.200 hectáreas, es decir, $37,45 \%$ de toda la superficie protegida en la entidad bajo el esquema de área natural protegida (INEGI ${ }^{9}, 2011$ ) decretado en 1978, ii) la REBIMA concentra aproximadamente $31 \%$ de las especies de mamíferos, $48 \%$ de las aves y $44 \%$ de las mariposas diurnas (De la Maza, 2006 , p. 10), iii) produce $30 \%$ del agua de las cuencas más importantes de México: Usumacinta-Grijalva, además, los ríos de 
esta reserva desembocan en el Lacantún y Usumacinta, llegando al mar cargados de nutrientes indispensables para las pesquerías del sur del Golfo de México (Carabias, Meli, Hernández y Almeida, 2006, p. 30). Concretamente las localidades de estudio fueron Lacanhá-Chansayab y San Javier que se encuentran dentro de la REBIMA, y Frontera Corozal, localidad ubicada fuera de la Reserva y colindante con Guatemala. Todas las localidades pertenecen al municipio de Ocosingo.

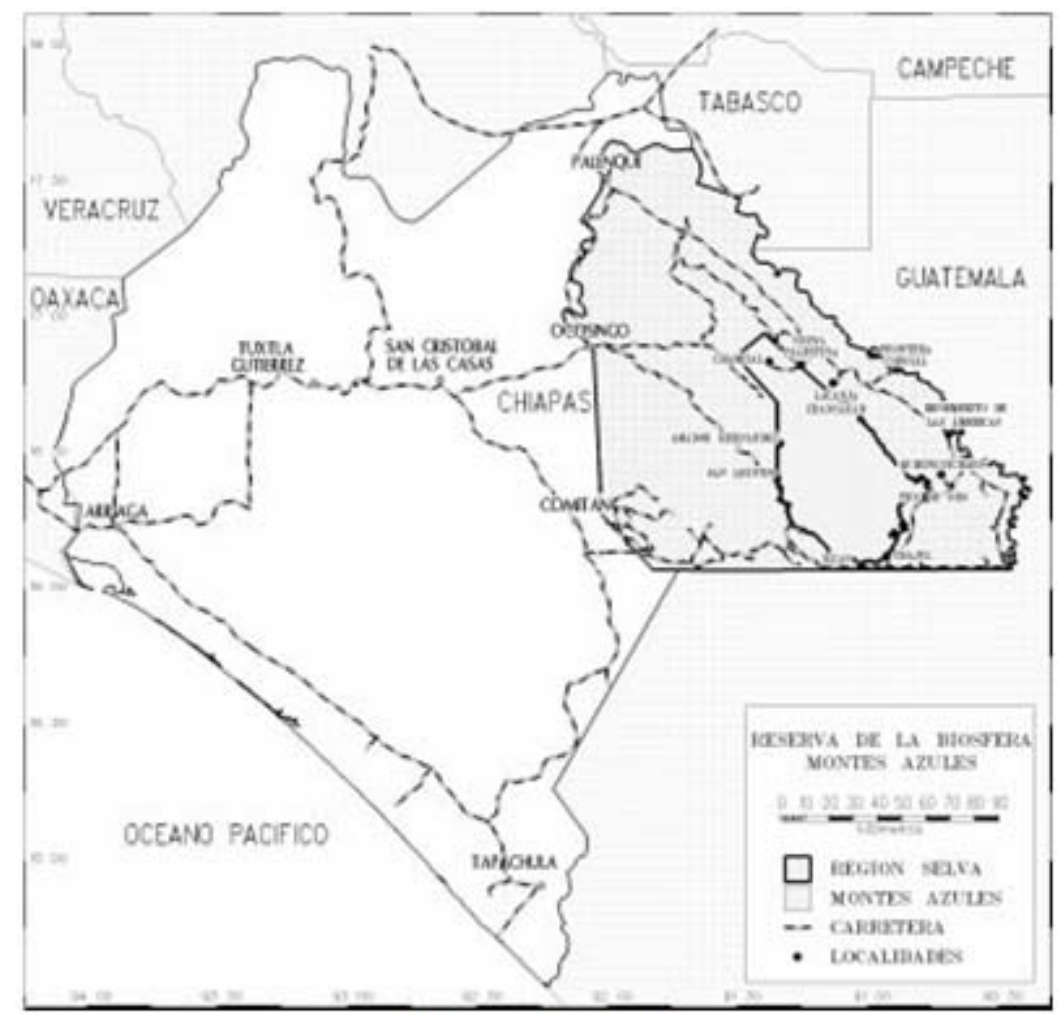

Fuente: INE (2000).

Figura 1. Región de la Selva Lacandona.

El estudio contempló como sujetos de estudio a una de las etnias representativas del estado de Chiapas, los lacandones, establecidos en las localidades de Lacanhá-Chansayab (dos entrevistas realizadas) y San Javier (una entrevista realizada). Los proveedores de servicios ecoturísticos entrevistados fueron Campamento Top Che, Campamento Yatoch Barum, y Centro Ecoturístico 3 Lagunas, respectivamente. En la localidad de Frontera Corozal, se encuentra establecida la etnia chol, grupo minoritario y en cierta forma segregada en el acceso a recursos públicos establecido fuera de la REBIMA, y no forma parte de la etnia lacandona, que es el grupo étnico favorecido por autoridades gubernamentales del estado de Chiapas. El proveedor de servicios entrevistado fue la Sociedad Cooperativa de Bienes y Servicios Nueva Alianza. Todos los entrevistados fueron elegidos por su propensión a compartir información y la disponibilidad para participar en el estudio. Las entrevistas se llevaron a cabo durante el 05 de septiembre al 25 de octubre de 2011. 


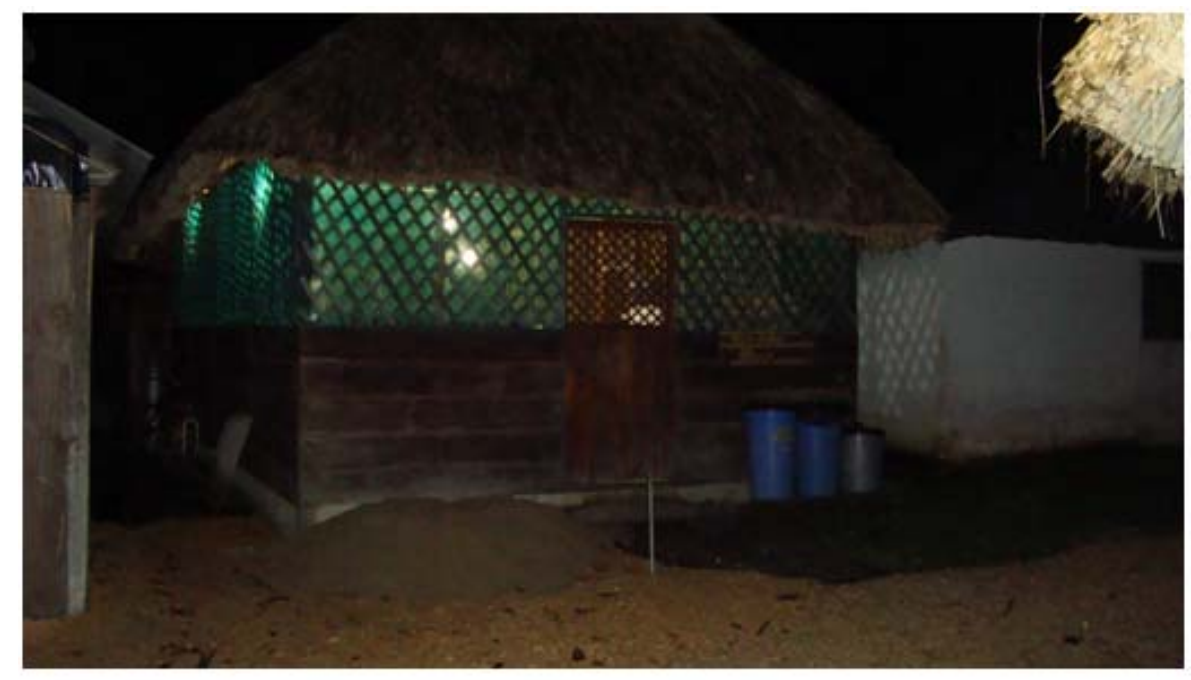

Imagen 1. Campamento Top Che.

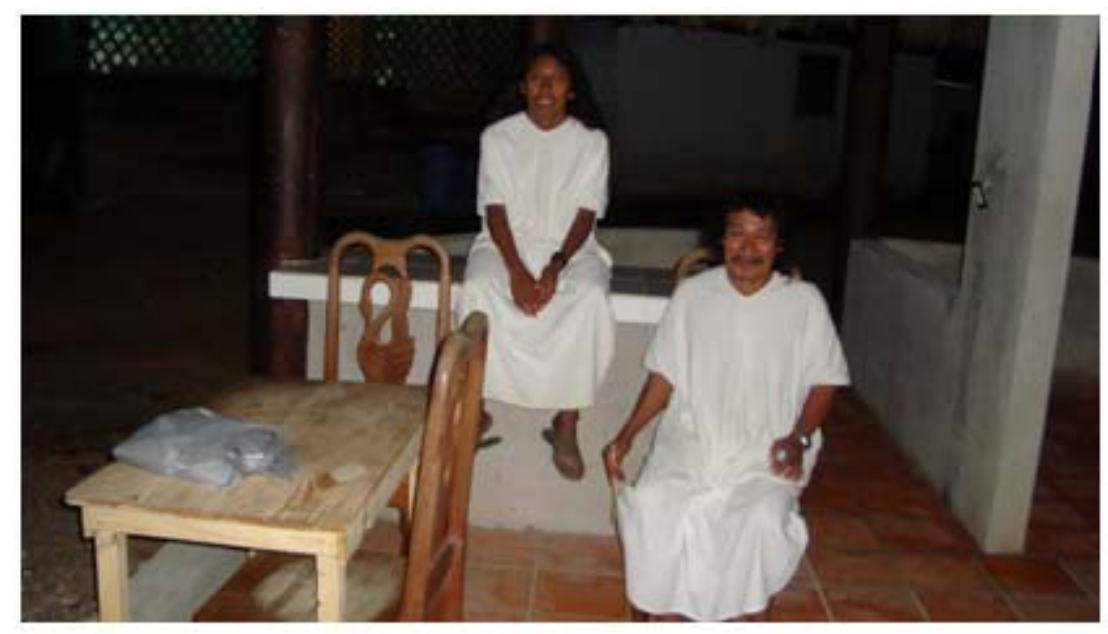

Imagen 2. Campamento Top Che. 


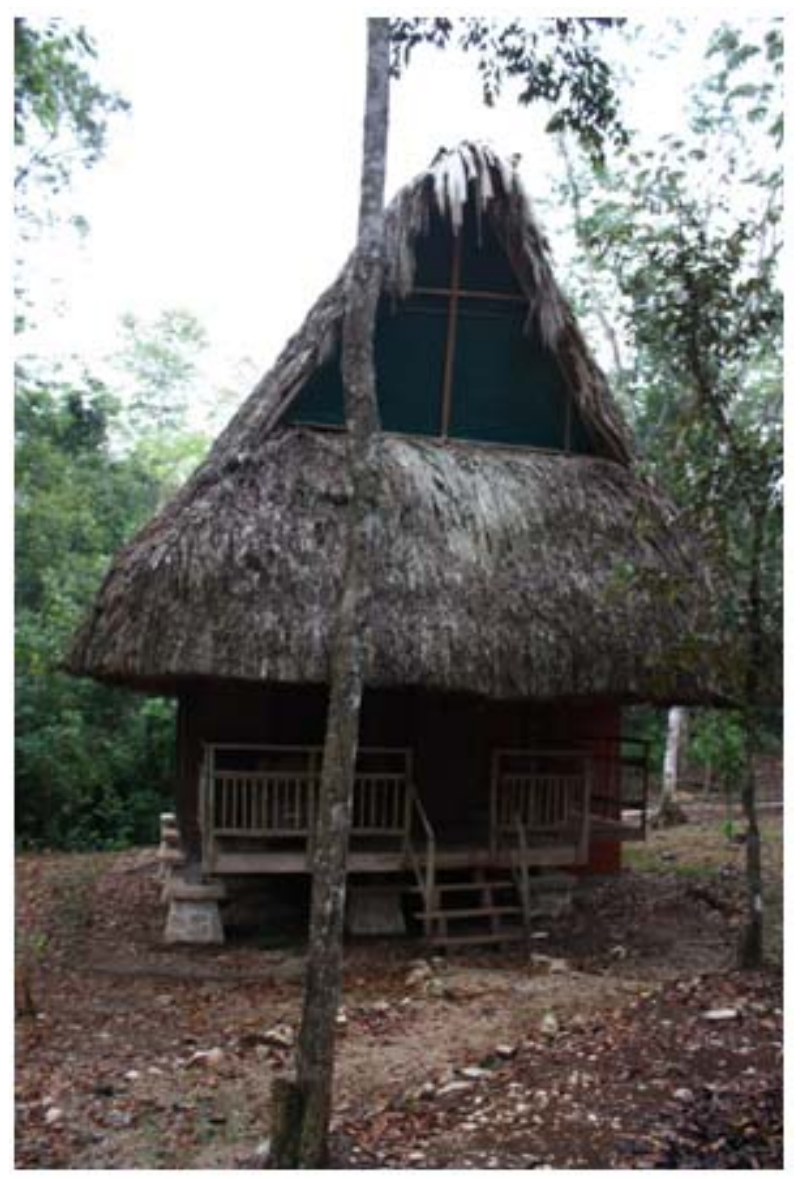

Imagen 3. Centro Ecoturístico 3 Lagunas. 


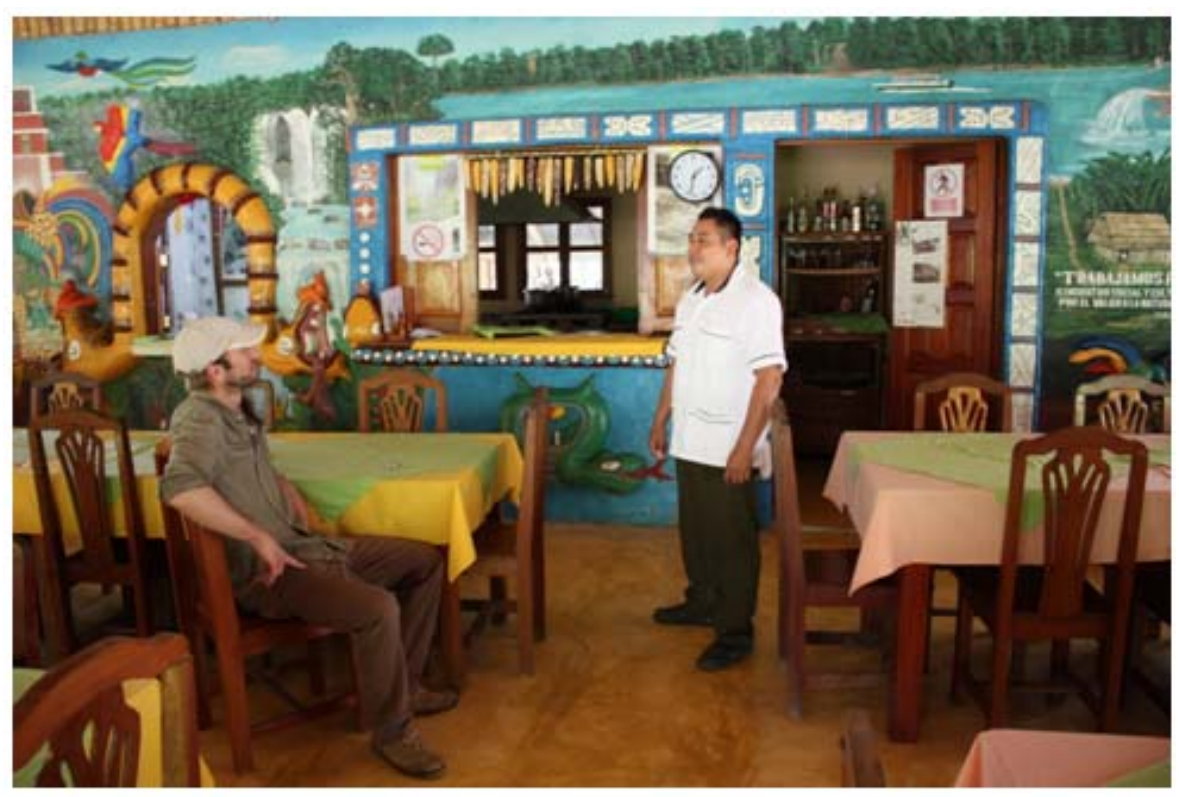

Imagen 4. Centro Ecoturístico Nueva Alianza Frontera Corozal.

Los apartados considerados fueron: (i) datos generales, en donde se indica nombre del entrevistado, datos de contacto, edad, municipio, entre otros; (ii) caracterización de la unidad económica y de la actividad ecoturística desempeñada, que refiere antigüedad de la empresa, tiempo transcurrido entre el diseño del proyecto ecoturístico y la puesta en marcha, superficie con la que cuenta el entrevistado, número de empleados, tipos de turistas recibidos, etcétera; (iii) desempeño económico de la empresa, solicitando ingresos y costos estimados de los años 2007 y 2011; (iv) índice de sustentabilidad ecoturística, referido a la integración de seis ejes: económicos, sociales, tecnológicos, institucionales, medioambientales y del entorno, que integra un total de 19 variables, que permitieron comparar el grado de sustentabilidad en el momento actual (2011) con respecto al 2007.

\section{Índice de Sustentabilidad Ecoturística}

La lógica y la formulación del índice se ubica, en términos conceptuales, en el marco de los métodos que integran los marcos de evaluación, para ello, se revisaron diversos documentos (incluyendo marcos normativos), especialmente las investigaciones de Rendón (2004), De Camino y Müller (1993) y OCDE (1993). EI panel de expertos se realizó del 15 al 22 de junio de 2011, y en el cual, participaron académicos, organizaciones no gubernamentales y algunos prestadores de servicios profesionales que laboran para dependencias de gobierno federal relacionadas con los recursos naturales. Algunas de las charlas y comentarios fueron realizadas vía Skype, correo electrónico y/o vía telefónica. 
Diagrama 1. Proceso de formulación e integración de las variables para valorar la sustentabilidad en espacios rurales relacionados con el ecoturismo en la Reserva de la Biosfera Montes Azules (REBIMA), Ocosingo, Chiapas, México

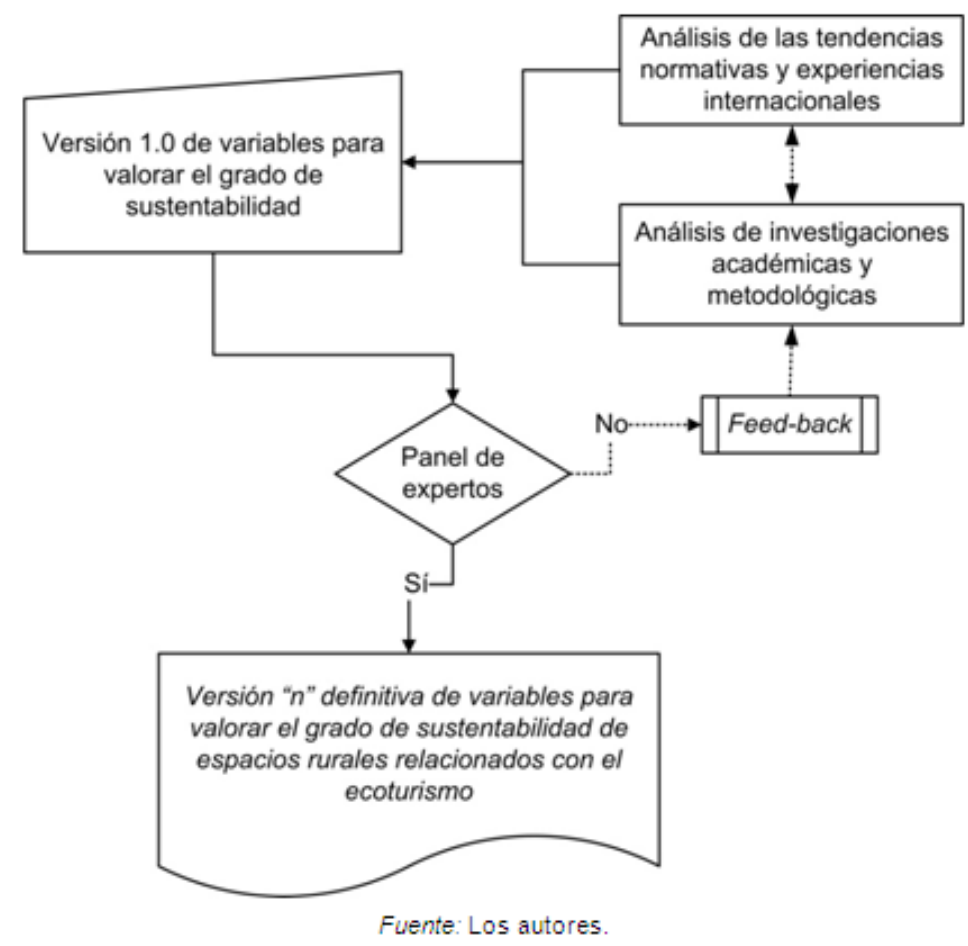

Las variables formuladas e integradas por eje temático fueron las siguientes:

1) Eje económico, contempla cuatro variables: i. Retribución económica de la empresa ecoturística; ii. Capacidad de absorber (interiorizar) pérdidas económicas; iii. Visión económica prospectiva (anticipación a las necesidades del mercado); iv. Relación beneficio/costo.

2) Eje social, integró tres variables: v. Acceso a servicios básicos (energía eléctrica, agua potable, drenaje y salud) en la empresa y en la vivienda del titular de la empresa; vi. Escolaridad del empresario titular; vii. Distribución de beneficios económicos.

3) Eje tecnológico, aborda dos variables: viii. Acceso y recepción de asesoría técnica más que consultoría; ix. Realización de vigilancia tecnológica que permite desarrollar y fortalecer capacidades locales.

4) Eje institucional, contempló cuatro variables: $x$. Organización formal (no grupos de trabajo) y participativa que promueve la formación de cuadros técnicos bajo una óptica de política social; xi. Cumplimiento de normas de regulación aplicables al ecoturismo; xii. Acceso a subsidios públicos y privados preferenciales por la condición étnica y/o por el carácter ecoturístico; xiii. Cumplimiento de mecanismos específicos de transparencia y rendición de cuentas en torno a los subsidios y/o financiamientos otorgados por solicitud de alguna institución pública y/o privada. 
5) Eje medioambiental, engloba a cuatro variables: xiv. Capacitación en aspectos clave de la llamada sustentabilidad social del ecoturismo para titulares de empresas y turistas; xv. Adopción de energías alternativas (energía solar, eólica y/o diseños bioclimáticos) en la dinámica operativa de la empresa; xvi. Adopción de sistemas optimizadores de agua (captación de agua pluvial, medidas y dispositivos ahorradores, reutilización del agua tratada y tratamiento de aguas residuales) en la dinámica operativa de la empresa; xvii. Adopción de sistemas de gestión de residuos sólidos de la mano con un programa de educación ambiental.

6) Eje del entorno, integrado por dos variables: xviii. Tendencia a la alza en el precio de bienes y servicios diferenciados que se ofertan en la empresa; xix. Posicionamiento competitivo favorable.

Todas las variables fueron medidas en escala ordinal y sirvieron de base para el diseño de un Índice de Sustentabilidad Ecoturística, indicando: 1 escenario deseable, 0,75 escenario con perspectiva de mejora, 0,5 escenario no deseable, considerando para ello, los datos e información compartida por los responsables de las empresas ecoturísticas entrevistadas, así como la valoración de un agente externo, en este caso el entrevistador y su experiencia en campo y en proyectos ecoturísticos.

Las variables: iv. Relación beneficio/costo, vi. Escolaridad del titular de la empresa, vii. Distribución de beneficios económicos, xviii. Tendencia a la alza en el precio de bienes y servicios diferenciados que se ofertan en la empresa, y xix. Posicionamiento competitivo favorable, se consideraron como compuestas dado que dependen de otros datos, tomando como base los aportes de Rendón (2004, pp. 119-142). Por ejemplo, en el caso de la relación beneficio/costo, la información se colectó con el apartado (iii) desempeño económico de la empresa, del instrumento de colecta, considerando los años 2007 y 2011. Los rangos de tal variable fueron determinados de la forma siguiente: escenario no deseable (1,9 o menos), escenario con perspectivas de mejora $(2,0-2,5)$ y escenario deseable $(2,6$ o más). La escolaridad se colectó en el apartado (i) datos generales y los rangos empleados para evaluarla fueron: 0,5 cuando el titular o administrador de la empresa no cuenta con estudios formales pero existe capacitación en el trabajo vía asesores o consultores, 0,75 se cuenta con estudios básicos completos (primaria o secundaria) y capacitación en el trabajo, y 1,0 cuando el responsable de la empresa posee educación media superior y superior.

En el caso de la variable vii. Distribución de beneficios económicos, se consideró la fórmula: (Utilidad / Monto destinado al pago de mano de obra) x 100; dicha información se colectó en el apartado (iii). Las variables: xviii. Tendencia a la alza en el precio de bienes y servicios diferenciados que se ofertan en la empresa, explora la tendencia de precios en el mercado nacional y regional. $\mathrm{Y}$ la variable xix. Posicionamiento competitivo favorable, se obtuvo con información del apartado (iii). En cuanto al asunto del posicionamiento competitivo, fue calculado con base en los aportes de Muñoz, Aguilar, Espejel, García y Rendón (2006, pp. 92-93) con la fórmula siguiente: $\Delta \%$ Ingresos $=((\Delta \%$ Ingresos $-\mathrm{Me}) /(\Delta \%$ IngresosMáx - Me)) x 100 ; donde: $\Delta \%$ Ingresos es el incremento 
porcentual en los ingresos, Me: es la mediana o valor medio de la serie de datos, $\Delta$ Ingresos se refiere al incremento en los ingresos, $\Delta$ IngresosMáx es el incremento de los ingresos máximos.

\section{Captura y análisis de la información}

La captura de la información se realizó en Microsoft Office Excel 2007, correspondiendo una hoja de cálculo por cada apartado del instrumento de colecta, mismas que fueron nombradas de la siguiente forma: (i) datos generales: DatGen, (ii) caracterización de la unidad económica y de la actividad ecoturística desempeñada: CaractEmp, (iii) desempeño económico de la empresa: DesempEco, (iv) índice de sustentabilidad ecoturística: ISE. Los datos fueron evaluados mediante el análisis de componentes principales Categórico (CATPCA, por sus siglas en inglés) con Escalamiento Óptimo, porque permite el estudio de variables cualitativas (nominal y ordinal), a través de niveles de escalamiento óptimo en lugar de asumir distribución de las muestras (Gifi, 1990). Meulman y Heiser $(2010$, p. 8) indican que la solución de un análisis de componentes principales categórico maximiza las correlaciones de las puntuaciones de objetos con cada una de las variables cuantificadas para el número de componentes (dimensiones) especificado.

Este análisis, se empleó para reducir la estructura de datos en un conjunto más pequeño de dimensiones no correlacionadas que representen la mayor parte de la información encontrada en las variables originales y clasifica las unidades de análisis en grupos. La técnica es más útil cuando un extenso número de variables impide una interpretación eficaz de las relaciones entre los objetos (sujetos y unidades). Al reducir la dimensionalidad, se interpreta un pequeño número de dimensiones en lugar de un extenso número de variables (Meulman y Heiser, 2010, p. 27). En torno a las correlaciones, Restrepo y González (2007, p. 185) indican que son útiles para medir el grado de relación o asociación existente entre dos variables aleatorias sin atribuir relaciones causales. En la presente investigación se empleó el coeficiente de correlación de Spearman, que es un coeficiente no paramétrico alternativo al coeficiente de correlación de Pearson, que requiere que las variables estén medidas, cuando menos una, en escala ordinal (Tomás-Sábado, 2009, p. 104). El software empleado para los análisis fue SPSS versión 19.0 y SAS versión 9.0 para Windows.

\section{HALLAZGOS Y RESULTADOS}

Tres de los cuatro entrevistados $(75 \%)$, proveedores de servicios ecoturísticos, son hombres. La edad promedio de los entrevistados es de 36 años, poseen siete años de escolaridad, es decir, primer año de educación secundaria concluido y primaria completa (educación básica), y cuentan con 11 años de experiencia en torno a proyectos ecoturísticos y/o empresas. Las empresas de los proveedores de servicios ecoturísticos entrevistados tienen en promedio ocho años de antigüedad, y cuando iniciaron su travesía en "tramitología" mexicana transcurrieron tres años entre el diseño y 
la puesta en marcha. La superficie promedio con la que cuentan es de aproximadamente 1,9 hectáreas. En promedio, $78 \%$ de los ingresos provienen de la actividad ecoturística, con un ingreso estimado para 2007 de $\$ 312.584,87$ (USD $\$ 24.615,50^{10}$ ) con un costo estimado de $\$ 167.801,50$ (USD $\$ 13.214,07$ ) y para 2011 ingresos de $\$ 434.572,93$ (USD\$ 34.221,84) con costos de $\$ 302.200,00$ (USD $\$ 23.797,71$ ), con una relación beneficio-costo promedio que ha venido a menos, pasando de 2,7 a 1,7, entre 2007 y

$75 \%$ de las empresas ecoturísticas (tres de cuatro) se encuentran certificadas por el cumplimiento de la NMX-AA-133-SCFI-2006 que aborda los requisitos y especificaciones de sustentabilidad del ecoturismo. En promedio, se reciben anualmente 109 turistas locales/regionales, 1.277 turistas nacionales y 968 turistas internacionales con presencia estacional muy marcada (diciembreenero, marzo-abril y julio-agosto). La generación promedio de empleos, asalariados y no asalariados, asciende a 11 vacantes por empresa ecoturística, de las cuales cinco vacantes son ocupadas por mujeres y seis por hombres, siete de las 11 vacantes son asalariadas y cuatro no asalariadas. El referente del pago mensual es el salario mínimo (\$59,08 equivalentes a USD\$ 4,65).

El principal canal de marketing que los empresarios utilizan es la propaganda institucional $(60 \%)$ seguida de las páginas web $(40 \%)$. Con todo y la situación actual y el entorno de crisis generalizada, la percepción de los empresarios $(75 \%)$ es que la empresa está creciendo y se espera que en un futuro la empresa continúe de esta manera (50\%). En promedio, el año 2010 es percibido como el año de mayor afluencia turística, mientras que 2008 como el de menor afluencia. En promedio, se reciben apoyos mensuales/anuales (75\%) provenientes de recursos públicos por un monto de $\$ 1 ' 209,000.00$ (USD $\$ 95.206,60$ ), al igual que, se reciben $(75 \%)$ 3.000 árboles para labores de reforestación por parte de la Comisión Nacional Forestal. La actividad secundaria se relaciona con la agricultura en el $75 \%$ de los casos (tres de cuatro empresarios), la cual proporciona en promedio $23 \%$ del ingreso, lo que equivale a un monto de $\$ 125.324,76$ (USD $\$ 9.869,10$ ), en tanto que $50 \%$ de las empresas (dos de cuatro), manifiesta como una de las principales necesidades el desarrollo/fortalecimiento de capacidades mediante procesos formativos in situ (consultoría/asesoría).

De acuerdo a los resultados del análisis multivariado, en los eigenvalores para cada iteración se obtuvieron cantidades arriba de $1(\geq 11,97)$, lo que hace conveniente el análisis para determinar el porcentaje de varianza. Además se presentó una adecuada consistencia interna con valores superiores a 0,7 (alfa de Cronbach para la dimensión 1 de 0,946, dimensión 2 de 0,824 y la combinación de las dos dimensiones de 0,993 ) y un porcentaje de varianza acumulado en las primeras dimensiones de $92,6 \%$. 
Cuadro 1. Aporte de las diferentes variables en las dimensiones en el análisis de componentes principales categórico (CATPCA, por sus siglas en inglés)

\begin{tabular}{|c|c|c|}
\hline \multirow[t]{2}{*}{ Variables } & \multicolumn{2}{|c|}{ Dimensiones } \\
\hline & 1 & 2 \\
\hline $\begin{array}{l}\text { Capacidad de absorber } \\
\text { (interiorizar) pérdidas } \\
\text { económicas }\end{array}$ & $-0,361$ & $-0,786$ \\
\hline $\begin{array}{l}\text { Escolaridad del empresario } \\
\text { titular }\end{array}$ & $-0,461$ & $-0,872$ \\
\hline $\begin{array}{l}\text { Cumplimiento de mecanismos } \\
\text { específicos de transparencia }\end{array}$ & 0,991 & 0,055 \\
\hline Acceso a servicios básicos & $-0,991$ & $-0,055$ \\
\hline $\begin{array}{l}\text { Tendencia a la alza en el precio } \\
\text { de bienes y servicios }\end{array}$ & 0,991 & 0,055 \\
\hline $\begin{array}{l}\text { Realización de vigilancia } \\
\text { tecnológica para fortalecer } \\
\text { capacidades locales }\end{array}$ & $-0,428$ & 0,838 \\
\hline $\begin{array}{l}\text { Capacitación en aspectos clave } \\
\text { de la llamada sustentabilidad } \\
\text { social del ecoturismo }\end{array}$ & $-0,428$ & 0,838 \\
\hline $\begin{array}{l}\text { Posicionamiento competitivo } \\
\text { favorable }\end{array}$ & $-0,991$ & $-0,055$ \\
\hline $\begin{array}{l}\text { Adopción de sistemas } \\
\text { optimizadores de agua en la } \\
\text { dinámica operativa de la } \\
\text { empresa }\end{array}$ & 0,991 & 0,055 \\
\hline $\begin{array}{l}\text { Acceso a subsidios } \\
\text { preferenciales por la condición } \\
\text { étnica y/o ecoturística }\end{array}$ & $-0,975$ & 0,226 \\
\hline $\begin{array}{l}\text { Retribución económica de la } \\
\text { empresa ecoturística }\end{array}$ & $-0,991$ & $-0,055$ \\
\hline $\begin{array}{l}\text { Distribución de beneficios } \\
\text { económicos }\end{array}$ & 0,361 & 0,786 \\
\hline $\begin{array}{l}\text { Cumplimiento de normas de } \\
\text { regulación aplicables al } \\
\text { ecoturismo }\end{array}$ & $-0,428$ & 0,838 \\
\hline
\end{tabular}

Fuente: Los autores.

El cuadro del aporte de los componentes muestra las relaciones entre las variables y la dimensión de la solución (Cuadro 1). Valores 
grandes $(>0,5)$ en cada dimensión, indican que la variable está fuertemente asociada con la dimensión referida. La primera dimensión separa con valores positivos las variables: Cumplimiento de mecanismos específicos de transparencia y rendición de cuentas en torno a los subsidios y/o financiamientos otorgados por solicitud de alguna institución pública y/o privada (eje institucional), Tendencia a la alza en el precio de bienes y servicios diferenciados que se ofertan en la empresa (eje del entorno) y Adopción de sistemas optimizadores de agua (captación de agua pluvial, medidas y dispositivos ahorradores, reutilización del agua tratada y tratamiento de aguas residuales) en la dinámica operativa de la empresa (eje medioambiental), mientras que de manera negativa el Acceso a servicios básicos (energía eléctrica, agua potable, drenaje y salud) en la empresa y en la vivienda del titular de la empresa (eje social), Posicionamiento competitivo favorable (eje del entorno) y Retribución económica de la empresa ecoturística (eje económico).

La segunda dimensión se relaciona positivamente con la Realización de vigilancia tecnológica que permite desarrollar y fortalecer capacidades locales (eje tecnológico), Capacitación en aspectos clave de la llamada sustentabilidad social del ecoturismo (incluida la educación ambiental) para titulares de empresas y turistas (eje medioambiental) y Cumplimiento de normas de regulación aplicables al ecoturismo (eje institucional) (DOF, 2006) ${ }^{11}$. De manera negativa interactúa con la Escolaridad del empresario titular (eje social) y la Capacidad de absorber (interiorizar) pérdidas económicas (eje económico).

Diagrama 2. Representación de las dos primeras dimensiones del CATPCA

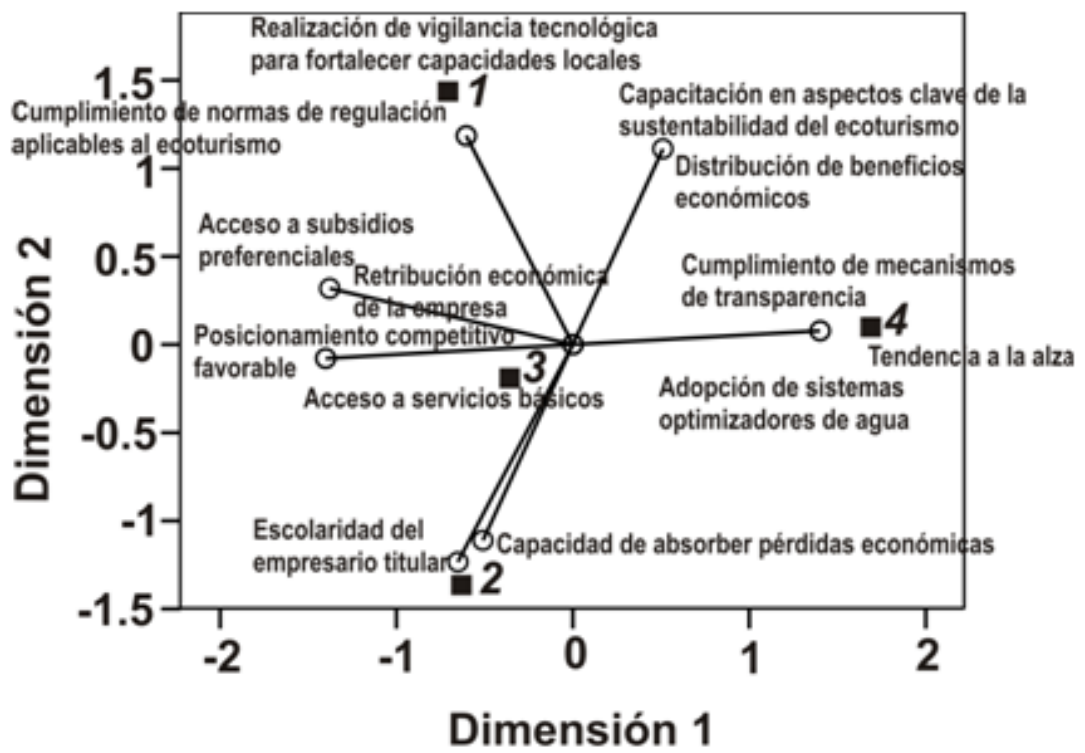

Fuente: Los autores.

En el Diagrama 2 se destacan aquellos proveedores de servicios que están más relacionados con alguna(s) variable(s). La dimensión 1 separa claramente a los proveedores de servicios que se encuentran dentro de la REBIMA y aquel que está fuera. Esto está muy relacionado no solo con el ámbito geográfico, sino también con 
aspectos culturales y administrativos. Particularmente la empresa número cuatro, se encuentra vinculada con la Tendencia a la alza en el precio de bienes y servicios diferenciados que se ofertan en la empresa (eje del entorno), Cumplimiento de mecanismos específicos de transparencia y rendición de cuentas en torno a los subsidios y/o financiamientos otorgados por solicitud de alguna institución pública y/o privada (eje institucional), y Adopción de sistemas optimizadores de agua (captación de agua pluvial, medidas y dispositivos ahorradores, reutilización del agua tratada y tratamiento de aguas residuales) en la dinámica operativa de la empresa (eje medioambiental). Esto es lógico en la medida en que por encontrarse fuera de la REBIMA, y por tanto, el acceso a subsidios se ve limitado (por no ser lacandones), así que cuando se reciben este tipo de subvenciones existe un mayor esfuerzo por comprobar y transparentar el uso de los recursos.

La dimensión 2 separa de manera importante a los proveedores de servicios que se encuentran en la REBIMA. Particularmente, al proveedor de servicios número 1 , una de las empresas establecidas dentro de la Reserva, fortalece la Realización de vigilancia tecnológica que permite desarrollar y fortalecer capacidades locales (eje tecnológico) y el Cumplimiento de normas de regulación aplicables al ecoturismo (eje institucional). De hecho, en buena medida su posicionamiento en el ámbito de los proveedores de servicios ecoturísticos en la región se explica por sus constantes innovaciones en el servicio, apertura a la participación de eventos formativos, constante formación de vínculos con agencias de viajes y demás transportadores y el cumplimiento de la normatividad aplicable NMX-AA-133-SCFI-2006. Contrario a lo que sucede con el proveedor número 2, empresa establecida en la Reserva, que presenta una mayor escolaridad (eje social) y tiene una mayor Capacidad de absorber (interiorizar) pérdidas económicas (eje económico), lo cual remite al principio de relación directamente proporcional, mayor escolaridad mayor capacidad de absorber pérdidas. Particularmente la titular del campamento no tiene ascendencia étnica -aunque su esposo es lacandón-, se desempeñó como profesional técnico especializado en turismo laborando para la Secretaría de Turismo en Oaxaca, México, y desde hace más de 10 años radica en la REBIMA y lleva las riendas de la empresa familiar.

El proveedor de servicios número 3, establecido en la Reserva, está más vinculado con el Acceso a subsidios preferenciales por la condición étnica y/o por el carácter ecoturístico (eje institucional), Retribución económica de la empresa ecoturística (eje económico), Posicionamiento competitivo favorable (eje del entorno) y Acceso a servicios básicos (eje social), es decir, depende primordialmente de los subsidios para permanecer en el mercado. En buena medida, su estrategia de servicios es masiva (turistas de mochila que pernoctan en literas).

En síntesis, el ranking de las empresas rumbo a la sustentabilidad, es decir, los mayores esfuerzos con miras alcanzar la sustentabilidad en el contexto del ecoturismo en la REBIMA son realizados por la empresa número 1 , en segundo lugar la empresa número 3 , posteriormente la empresa número 4 y finalmente la empresa número 2. 


\section{CONSIDERACIONES FINALES}

La presente investigación presenta una propuesta metodológica para evaluar el grado de sustentabilidad en espacios rurales relacionados con el ecoturismo, mediante el diseño de un índice de sustentabilidad ecoturística basado en la integración de seis ejes: económicos, sociales, tecnológicos, institucionales, medioambientales y del entorno. Sin embargo, hay que reconocer las limitaciones del estudio, sobre todo considerando el número empresas y titulares de las mismas entrevistados: tres empresas dentro de la Reserva de la Biosfera Montes Azules (REBIMA), y una empresa fuera de la REBIMA, ubicadas todas en el municipio de Ocosingo, Chiapas, México. La principal limitante observada en el presente estudio, se encuentra en el número reducido de empresas dispuestas a proporcionar datos y facilidades para realizar la evaluación comparativa. Por cierto, las dos dimensiones presentadas explican poco más del $90 \%$ de la varianza.

Las empresas entrevistadas están lejos del cumplimiento del precepto de sustentabilidad visto desde una óptica integral, como el planteado en la actual investigación, en el contexto del ecoturismo en la REBIMA; sin embargo, se aprecian diversos esfuerzos que en mayor o menor medida las ubican en dicho camino. El ranking de las empresas rumbo a la sustentabilidad, es decir, los mayores esfuerzos con miras alcanzar la sustentabilidad son realizados por la empresa número 1 , en segundo lugar la empresa número 3 (ambas establecidas en la REBIMA), posteriormente la empresa número 4 (establecida fuera de la REBIMA) y finalmente la empresa número 2 (establecida en la REBIMA). La evidencia recabada indica un desequilibrio en el acceso a subsidios, donde la pertenencia étnica desempeña un rol trascendental.

Con miras a favorecer el trayecto emprendido por las empresas estudiadas, y otras más establecidas en los espacios rurales de América Latina, en el marco de la sustentabilidad, se reconoce que una ruta crítica pudiera considerar los siguientes aspectos: 1) Favorecer la sistematización y documentación de actividades relacionadas con ingresos y gastos de la empresa, pues el impacto de lo anterior se refleja en variables del eje económico. 2) Fortalecer el desarrollo de capacidades locales en materia de educación ambiental para los titulares de las empresas y de estos con los turistas de la mano con sistemas de gestión de residuos sólidos eficientes y adecuados. 3) Más allá de la participación financiadora de las entidades gubernamentales del estado de Chiapas, México, es requerida la documentación de viabilidad y difusión, si fuera el caso, de diversas fuentes de energías alternativas (energía solar, eólica y/o diseños bioclimáticos) y de sistemas optimizadores de agua (captación de agua pluvial, medidas y dispositivos ahorradores, reutilización del agua tratada y tratamiento de aguas residuales).

Para lograr incidir en las actividades referidas, es necesario un proceso de acompañamiento/seguimiento realizado por equipos interdisciplinarios de profesionistas con arraigo y reconocimiento 
local comprometidos con la mejora de la calidad de vida y de la conservación/manejo de los recursos naturales que proporciona el espacio rural donde se ubica la REBIMA; sin embargo, y acorde con lo visto en campo, los esfuerzos realizados por entidades gubernamentales estatales y federales radica en la proveeduría de servicios de consultoría de empresas externas con trabajos en múltiples países de América Latina, quienes integran equipos de trabajo con pasantes y recién egresados de carreras como gastronomía, comunicaciones y licenciados en administración de empresas, que poco conocen sobre la realidad de la REBIMA y durante su estancia en dicho espacio (en promedio de 10 días) lograr disfrutar de periodos vacacionales considerables, pues no poseen experiencia en el manejo de grupos ni en estudios socioambientales, carecen de una formación intercultural y de técnicas y herramientas que incorporen enfoques y componentes andragógicos que puedan plasmarse en planes de formación que coadyuven al desarrollo de capacidades in situ y en beneficio de la sustentabilidad.

Si bien existe conocimiento especializado y se cuenta con recomendaciones sustanciales (UNDP, 2011) que proveen un marco integral para el desarrollo de la actividad turística con orientación hacia sustentabilidad, todavía existen vacíos institucionales sobre cómo erradicar la pobreza, incorporar la perspectiva de género y la participación comunitaria, e implementar estrategias que sean operativas en contextos locales, dado que se carece de estudios socioterritoriales que incorporen indicadores de sustentabilidad articulados al desempeño de la actividad turística.

\section{AGRADECIMIENTOS}

(i) Apoyos Complementarios para la Consolidación Institucional de Grupos de Investigación, modalidad II (Retención), Consejo Nacional de Ciencia y Tecnología: CONACYT (México). Proyecto auspiciado: "Sistema de evaluación y seguimiento de la política pública ecoturística en Chiapas", solicitud 145819, convocatoria 2010-02. (ii) Comisión de Operación y Fomento de Actividades Académicas (COFAA) y Programa de Estímulos al Desempeño de los Investigadores (EDI), Instituto Politécnico Nacional.

\section{REFERENCIAS}

- BANXICO. (2012). Mercado cambiario. Tipos de cambio para solventar obligaciones denominadas en dólares de los EE.UU.A. pagaderas en la República Mexicana. Recuperado el 5 de octubre de 2012 de http://www.banxico.org.mx/portal-mercadocambiario/index.html

- Carabias, J., Meli, P., Hernández, G. y Almeida, G. (2006). Estrategia de restauración ambiental y prevención de incendios en ejidos ribereños del río Lacantún colindantes a la Reserva de la Biosfera Montes Azules (Reporte Final). México, D.F.: Instituto 
Nacional de Ecología A.C. y Universidad Nacional Autónoma de México.

- De Camino, V.R. y Müller, S. (1993). Sostenibilidad de la agricultura y los recursos naturales: bases para establecer indicadores. Serie Documentos de Programas 38. San José, Costa Rica: Instituto Interamericano de Cooperación para la Agricultura (IICA).

- De la Maza, J. (2006). Montes Azules, un patrimonio natural de México y del mundo. Impulso Ambiental, Revista de divulgación e información, 33: 9-13.

- DOF. (2006). NMX-AA-133-SCFI-2006. Requisitos y especificaciones de sustentabilidad del ecoturismo. Diario Oficial de la Federación (DOF), México, D.F.

- Galván, Y., Masera, O. y López, S. (2008). Las evaluaciones de sustentabilidad. En Astier, M., Masera, O. y Galván, Y. (Coords.), Evaluación de sustentabilidad. Un enfoque dinámico y multidimensional. Sociedad Española de Agricultura Ecológica (SEAE) / Centro de Investigaciones en Geografía Ambiental (CIGA) / El Colegio de la Frontera Sur (ECOSUR) / Centro de Investigaciones en Ecosistemas (CIEco) / Universidad Nacional Autónoma de México (UNAM) / Grupo Interdisciplinario de Tecnología Rural Apropiada A.C. (GIRA) / Mundi-Prensa México, S.A. de C.V. / Fundación Instituto de Agricultura Ecológica y Sustentable, Valencia.

- Gifi, A. (1990). Nonlinear multivariate analysis. Chichester, West Sussex: John Wiley \& Sons.

- Halffter, G. (2011). Reservas de la biosfera: problemas y oportunidades en México. Acta Zoológica Mexicana (n.s.), 27(1): 177-189.

- Hernández, S.L., López, L. y Pons, J.M. (2006). Turismo: ¿La industria sin chimeneas? En Aguilar, P. y Pons, J.M. (Coords.), Saber para proteger. Introducción al ecoturismo comunitario. México, D.F.: Centro de Educación y Capacitación para el Desarrollo Sustentable, Secretaría de Medio Ambiente y Recursos Naturales (SEMARNAT) y Comisión Nacional Forestal (CONAFOR).

- INE. (2000). Programa de Manejo Reserva de la Biosfera Montes Azules. Instituto Nacional de Ecología INE / Secretaría de Medioambiente Recursos Naturales y Pesca SEMARNAP, México, D.F.

- INEGI. (2011). Biodiversidad. Áreas Naturales Protegidas con Programa de Manejo, 2011. Recuperado el 4 de septiembre de 2012 de

http://www.inegi.org.mx/sistemas/sisept/default.aspx?t=mamb14 $0 \& c=21468 \& s=e s t$

- Ivars, J. A. (2000). Turismo y espacios rurales. Conceptos, filosofía y realidades. Revista Investigaciones Geográficas, 023: 59-88.

- Jules, N.P. (1995). Participatory learning for sustainable agriculture. World Development, 23(8): 1247-1263.

- Leyva Solano, X. y Ascencio, G.F. (1996). Lacandonia al filo del agua. Chiapas, México: Edit. Fondo Cultura Económica.

- Masera, O., Astier, M., López-Ridaura, S., Galván-Miyoshi, Y., OrtizÁvila, T., García-Barrios, L.E., García-Barrios, R., González, C. y Speelman, E. (2008). El proyecto de evaluación de sustentabilidad MESMIS. En Astier, M., Masera, O. y Galván, Y. (Coords.), Evaluación de sustentabilidad. Un enfoque dinámico y multidimensional. Sociedad Española de Agricultura Ecológica (SEAE) / Centro de Investigaciones en Geografía Ambiental (CIGA) / El Colegio de la Frontera Sur (ECOSUR) / Centro de Investigaciones 
en Ecosistemas (CIEco) / Universidad Nacional Autónoma de México (UNAM) / Grupo Interdisciplinario de Tecnología Rural Apropiada A.C. (GIRA) / Mundi-Prensa México, S.A. de C.V. / Fundación Instituto de Agricultura Ecológica y Sustentable, Valencia.

- Meulman, J.J. y Heiser, W.J. (2010). IBM SPSS Categories 19. Manual del usuario del sistema básico. Armonk, New York: IBM.

- Muñoz, M., Aguilar, J., Espejel, A., García, J.G. y Rendón, R. (2006). Informe de evaluación nacional: Subprograma de Investigación y Transferencia de Tecnología. México, D.F.: Secretaría de Agricultura, Ganadería, Desarrollo Rural, Pesca y Alimentación.

- Navarrete, D. y Halffter G. (2008). Dung beetle (Coleoptera: Scarabaeidae: Scarabaeinae) diversity in continuous forest, forest fragments and cattle pastures in a landscape of Chiapas, Mexico: the effects of anthropogenic changes. Biodiversity Conservation, 17: 2869-2898.

- OCDE. (1993). Core set of indicators for environmental performance reviews: a synthesis report by the group on the state of the environment. Environment Monographs OCDE / GD (93)179, Organization for Economic Cooperation and Development (OCDE), Paris.

- Organización Mundial de Turismo -OMT-. (2014). Comunicado de Prensa: El turismo internacional supera las expectativas con 52 millones llegadas adicionales en 2013. Recuperado el 10 de marzo de 2014 de http://media.unwto.org/es/press-release/2014-01-20/ el-turismo-internacional-supera-las-expectativas-con-52millones-llegadas-a

- Rendón, R. (2004). Evaluación comparativa de sustentabilidad en sistemas agrícolas convencionales, mixtos y orgánicos de México. Tesis doctoral, Centro de Investigaciones Económicas, Sociales y Tecnológicas de la Agroindustria y la Agricultura Mundial, Universidad Autónoma Chapingo, Texcoco, Estado de México.

- Restrepo, L.F. y González, J. (2007). De Pearson a Spearman. Revista Colombiana de Ciencias Pecuarias, 20: 183-192.

- Reyes, M.E. (2004). Política agraria en Chiapas: atención a focos rojos. Revista de Estudios Agrarios, 26, 55-93.

- SECTUR. (2004). Guía de apoyos federales para proyectos de ecoturismo. México, D.F.: Secretaría de Turismo (SECTUR).

- TIES. (1990). What is ecotourism? Recuperado el 22 de septiembre de 2012 de http://www.ecotourism.org/what-is-ecotourism

- Tomás-Sábado, J. (2009). Fundamentos de bioestadística y análisis de datos para enfermería. Colección Infermeria trivium 2. Barcelona: Universidad Autónoma de Barcelona, Infermeria ecoles universitàries gimbernat.

- United Nations -UN-. (2010). United Nations Conference on Trade and Development: Proposals stemming from the International Highlevel Meeting of Experts on Sustainable Tourism for Development in the Least Developed Countries Caen, France, 12-14 October 2010. Recuperado el 16 de enero de 2014 de http://dtxtq4w60xqpw.cloudfront.net/sites/all/files/ pdf/unctad_caen_recommendations_oct2010_en.pdf

- United Nations Development Programme -UNDP-. (2011). Discussion Paper: Tourism and Poverty reduction Strategies in the integrated Framework for Least developed Countries. Recuperado el 31 de diciembre de 2013 de http://dtxtq4w60xqpw.cloudfront.net/sites/all/files/pdf/ undp_discussion_paper_tourism_and_poverty_reduction_strate 
gies_in the

integrated_framework_for_least_developed_countries.pdf

- WTTC. (2012a). Travel \& tourism: World economic impact report 2012. Recuperado el 20 de septiembre de 2012 de http://www.wttc.org/site_media/uploads/downloads/world2012.p df

- (2012b). Travel \& tourism: Latin America economic impact report 2012. Recuperado el 20 de septiembre de 2012 de http://www.wttc.org/site_media/uploads/downloads/ latin_america2012.pdf

- (2012c). Travel \& tourism: Mexico economic impact report 2012. Recuperado el 20 de septiembre de 2012 de http://www.wttc.org/site_media/uploads/downloads/ mexic02012.pdf

- . (2012d). Travel \& tourism: Costa Rica economic impact report 2012. Recuperado el 20 de septiembre de 2012 de http://www.wttc.org/site_media/uploads/downloads/ costa_rica2012.pdf

- . (2012e). Travel \& tourism. Argentina economic impact report 2012. Recuperado el 20 de septiembre de 2012 de http://www.wttc.org/site_media/uploads/downloads/ argentina2012.pdf

1. Artículo de investigación producto del Proyecto "Sistema de evaluación y seguimiento de la política pública ecoturística en Chiapas, México", con financiamiento de los Apoyos Complementarios para la Consolidación Institucional de Grupos de Investigación, Consejo Nacional de Ciencia y Tecnología CONACYT (México), solicitud 145819, convocatoria 2010-02.

2. Doctor en Problemas Económico-Agroindustriales. ProfesorInvestigador del Centro Interdisciplinario de Investigación para el Desarrollo Integral Regional (CIIDIR) Oaxaca, Instituto Politécnico Nacional IPN, México. alberto.zarazua@gmail.com

3. Doctor en Antropología. Profesor-Investigador de El Colegio de Veracruz (COLVER).dmazabel@gmail.com

4. Doctor en Ecología Acuática. Profesor-Investigador del Centro Interdisciplinario de Investigación para el Desarrollo Integral Regional (CIIDIR) Michoacán, Instituto Politécnico Nacional IPN. rmoncayo@ipn.mx

5. Doctor en Problemas Económico-Agroindustriales. ProfesorInvestigador del Centro de Investigaciones Económicas, Sociales y Tecnológicas de la Agroindustria y la Agricultura Mundial (CIESTAAM), Universidad Autónoma Chapingo. redes.rendon@gmail.com

6. Los pronósticos para el desarrollo de la actividad turística son alentadores, especialmente en lo que respecta al turismo internacional. Particularmente propicio en el año 2013, da buenas señales para el presente año 2014 (Organización Mundial de Turismo, 2014). No obstante para el caso mexicano, habrá que considerar los impactos negativos que pueda ocasionar en el sector, la inusitada escalada de violencia que se ha instaurado en diversos estados del territorio nacional.

7. The International Ecotourism Society.

8. Secretaría de Turismo de México. 
9. Instituto Nacional de Estadística, Geografía e Informática. Es un organismo autónomo del gobierno mexicano encargado del Sistema Nacional de Información Estadística y Geográfica del país.

10. Las conversiones de pesos mexicanos a dólares americanos fueron calculadas con el tipo de cambio FIX determinado por el Banco de México (BANXICO) -banco central del Estado Mexicanocorrespondiente al 05 de octubre de 2012, equivalente a 12,6987 pesos por dólar. El cambio FIX es un promedio de las cotizaciones del mercado de cambios al mayoreo para operaciones liquidables el segundo día hábil bancario siguiente. Dichas cotizaciones se obtienen de plataformas de transacción cambiaria y otros medios electrónicos con representatividad en el mercado de cambios (BANXICO, 2012).

11. Diario Oficial de la Federación. Se trata del periódico oficial del Gobierno Constitucional de México. Su función es la publicación de leyes, reglamentos, acuerdos, circulares, órdenes y demás actos expedidos por los poderes de la Federación, a fin de que estos sean observados y aplicados debidamente en sus respectivos ámbitos de competencia en el territorio nacional.

Para citar este artículo: Zarazúa, J.A., Mazabel, D., MoncayoEstrada, R. \& Rendón-Medel, R. (2015). Evaluación de la sustentabilidad en espacios rurales relacionados con el ecoturismo. Estudio de caso en la Selva Lacandona, Chiapas, México. Revista Luna Azul, 40, 47-68. Recuperado de http://lunazul.ucaldas.edu.colindex.php?option=content\&task=v iew\&id=999 